\title{
Prospective prediction of suicide in a nationally representative sample: religious service attendance as a protective factor ${ }^{\dagger}$
}

\author{
Evan M. Kleiman and Richard T. Liu
}

\section{Background}

Previous research into religious service attendance as a protective factor against suicide has been conducted only retrospectively, with psychological autopsy studies using proxy informants of completed suicide, rather than prospectively, with completed suicide as a dependent variable.

\section{Aims}

To determine whether individuals who frequently attended religious services were less likely to die by suicide than those who did not attend so frequently.

\section{Method}

We analysed data from a nationally representative sample $(n=20014)$, collected in the USA between 1988 and 1994, and follow-up mortality data from baseline to the end of 2006.

\section{Results}

Cox proportional hazard regression analysis indicated that those who frequently attended religious services were less likely to die by suicide than those who did not attend, after accounting for the effects of other relevant risk factors.

\section{Conclusions}

Frequent religious service attendance is a long-term protective factor against suicide.

\section{Declaration of interest}

None.
Despite the well-documented link between religiousness and suicidality, ${ }^{1-4}$ no study to date has measured any dimension of religiousness as a prospective protective factor against completed suicide. Prior epidemiological studies have found that people with no religious affiliation were more likely to have a lifetime history of a suicide attempt than those who had a strong religious affiliation. $^{5-8}$ Although these epidemiological studies reinforced the link between religiousness and suicide, they examined only suicide attempts, usually based on retrospective or lifetime reports. The only study to date to examine religious service attendance in relation to death by suicide used retrospective proxy reports (psychological autopsy) for a sample of individuals aged 50 years and older, and found that the odds of having participated in religious activities throughout their lifetime were higher among those who died from natural causes compared with those who died by suicide. ${ }^{4}$ This study was limited by its sample and by its retrospective, psychological autopsy methodology. We present the first study to examine religious services attendance as a prospective protective factor against death by suicide. Specifically, we used first-person reports of potential risk and protective factors ascertained directly from individuals while they were alive in relation to prospectively occurring deaths by suicide over a 12- to 18-year period. Our prospective methodology eliminated reporting biases that might be associated with retrospective proxy informant reports. Using data from a large epidemiological study in the USA together with mortality follow-up data, allowing prospective prediction of actual death by suicide using survival analysis, we predicted that individuals who frequently attended religious services were less likely to die by suicide than those who did not attend. We considered other risk factors for suicide such as male gender, frequent alcohol and drug use, mood disorders and previous suicidal thoughts and behaviours. ${ }^{2-7}$ We also considered variables that could be confounded with religious service attendance, such as education level and socioeconomic

†See editorial, pp. 254-255, this issue. status, and other relevant social variables such as family size and frequency of time with friends. This study represents an advance on previously reported research in that it provides the first longitudinal prospective examination of protective factors against death by suicide rather than suicidal ideation or attempts. This is important because a relatively small proportion of those who attempt suicide actually die by that means, and this may in part be because of differences in intent and lethality of means. ${ }^{8,9}$ Thus, those who die by suicide may be qualitatively different from those who attempt suicide or have suicidal ideation. ${ }^{10}$

\section{Method}

Baseline data for the study came from the Third National Health and Nutrition Examination Survey (NHANES III) in the USA. ${ }^{11}$ This survey recorded data from a nationally representative sample of 39965 individuals over 2 months old, collected by the National Center for Health Statistics in two stages from 1988 to 1994 (1988-1991 and 1991-1994). All data, with the exception of mortality status, were collected at one time point only. Only participants aged 18 years or older $(n=20050)$ were interviewed by trained lay interviewers in mobile examination centres or in their home. These interviews consisted of demographic questions (e.g. gender and marital status), social functioning questions (e.g. how often one visits friends or attends religious services) and psychiatric history questions (e.g. previous diagnosis of depression). As these interviews contained the variables relevant to our study, our sample was limited to the 20014 out of 20050 persons who participated in the interviews and for whom complete data were available (99.82\%). Additional information on the method of data collection and weighting procedures is available elsewhere. ${ }^{11}$

Follow-up data came from the NHANES III linked mortality files that involved mortality data matched to the NHANES participants from the National Death Index (NDI) of the US 
Government Centers for Disease Control and Prevention (CDC) for individuals aged 18 years and over. All US States are required to submit electronic records of death along with causes of death to the division of the CDC that maintains the NDI database. When there is a discrepancy or lack of clarity in the cause of death information, a nosologist reviews the case to determine the actual cause of death. The NDI has been found to be highly accurate: one study demonstrated high sensitivity $(98.5 \%$ of deceased participants were correctly matched as dead) and high specificity (99.5\% of living participants were correctly matched as alive). ${ }^{12}$ Another study found $94.7 \%$ agreement between the NDI and the Social Security Death Index, another independent measure of US mortality status. ${ }^{13}$ The NDI search was conducted up to and including the last day of 2006 and included both mortality status and date of death in months after the initial interview (thus the maximum time frame was 216 months). A total of 20014 eligible participants $(100 \%)$ had mortality data or were believed to be alive (i.e. had no mortality data in the NDI in 2006). Of these, 25 individuals (unweighted) were known to have died by suicide between their initial interview session and 31 December 2006.

\section{Measures}

Demographic and social variables

All participating adults were asked a variety of questions about family demographics and social functioning. This included items on household size and how many times an individual talked to friends on the telephone (per week), spent time with friends locally (per year) and visited with friends out of town (per year).

\section{Socioeconomic status}

We used the poverty index as a measure of socioeconomic status. This is the ratio of a family's income to the poverty threshold (minimum family income for a given family size to indicate poverty) of the year the data were collected. This ratio allows the comparison of incomes from year to year, as data were collected across several years and the poverty level changed annually. The variable was coded so that higher scores represented a higher level of income relative to the poverty threshold.

\section{Alcohol and drug use}

Individuals who were frequent alcohol drinkers were identified by the question, "Was there ever a time or times in your life when you drank five or more drinks of any kind of alcoholic beverage almost every day?' Individuals who endorsed marijuana use more than 100 times were labelled as frequent marijuana users and individuals who used cocaine more than 10 times were labelled as frequent cocaine users.

\section{Lifetime psychiatric diagnoses}

Lifetime dysthymic disorder and bipolar disorder (types 1 and 2) diagnoses were determined using the National Institute of Mental Health Diagnostic Interview Schedule (DIS). ${ }^{14}$ This assessment was conducted by trained interviewers and is based on the DSM-III criteria for mental disorders. ${ }^{15}$ We were unable to assess depression diagnosis as a predictor variable. For depression to be included in statistical models using survival analysis, at least some of the participants who met diagnostic criteria for this disorder at baseline must also have experienced the prospective outcome of interest (i.e. suicide), which was not observed in our study.

\section{Suicidal ideation and suicide attempts}

Histories of suicidal ideation and attempts were assessed by interviewer questions from the DIS: 'Have you ever felt so low you thought of committing suicide?' and 'Have you ever attempted suicide?'

\section{Frequency of religious service attendance}

Frequent $v$. infrequent religious service attendance was assessed with the item 'How often do you attend church or religious services? (per year)'. Continuous data for this variable significantly violated assumptions of normality (skewness 20.78 , s.e. $=0.02$, kurtosis 437.59 , s.e. $=0.04)$. As transforming this variable did not appreciably improve the distribution, it was dichotomised so that frequent attender status was defined as attending religious services 24 times or more per year (i.e. approximately half of weekends per year). We chose 24 times or more per year as the cut-off for several reasons. First, this cut-off point has been used in prior studies with the NHANES III data-set; ${ }^{16}$ second, as noted in previous research, ${ }^{16}$ there was a strong preference among participants towards endorsing multiples of 12. Most participants endorsed attendance as zero, monthly (i.e. 12 times per year), twice-monthly (i.e. 24 times per year) or weekly (i.e. 48-52 times per year). Using 24 times per year as the cut-off, $58 \%$ of the sample were classified as frequent attenders. Using a cut-off of 12 was overly inclusive (63\% of the sample met this cut-off) and using a cut-off of 48 was overly exclusive ( $10 \%$ of the sample met this cut-off). Thus, using 24 times per year as the cut-off allowed us to avoid being either overly inclusive or exclusive.

\section{Statistical analysis}

We first conducted a series of univariate Cox proportional hazard regression models with each putative covariate predicting time to death by suicide. All predictors that were significant at $P \leqslant 0.10$ were entered into a multivariate Cox proportional hazard regression model. We weighted the data for this analysis based on the standard weighting guidelines for the NHANES data-set. ${ }^{17}$ All analyses were conducted using the complex samples module in SPSS version 21.0 for Mac.

\section{Results}

Table 1 lists unweighted descriptive statistics for all variables by mortality group. Table 2 lists the results of univariate Cox proportional hazard regression analyses using sample weights predicting time to death by suicide. Significant predictors at $P \leqslant 0.10$ were male gender (Wald $\chi^{2}=5.23$, hazard ratio $(\mathrm{HR})=5.11, P<0.05)$, age at baseline interview (Wald $\chi^{2}=3.00$, $\mathrm{HR}=0.97, P<0.10$ ), frequent marijuana use (Wald $\chi^{2}=5.86$, $\mathrm{HR}=6.18, \quad P<0.05$ ) and religious service attendance (Wald $\left.\chi^{2}=5.26, \mathrm{HR}=0.23, P<0.05\right)$. Specifically, male gender, older age at baseline interview and frequent marijuana use each predicted shorter time to death by suicide, whereas frequent religious service attendance predicted longer time to suicide. Table 3 lists the results of the multivariate Cox proportional hazard regression analysis predicting time to death by suicide and including the significant predictors from the first set of univariate analyses. Although a past suicide attempt was not a significant predictor of death by suicide, we still controlled for it in the final analysis given the strong link between past and future suicidal behaviour. ${ }^{15}$ Only male gender (Wald $\chi^{2}=4.94, \mathrm{HR}=3.69$, $P<0.05$ ) and religious service attendance (Wald $\chi^{2}=3.68$, $\mathrm{HR}=0.33, P<0.05)$ remained significant predictors of time to death by suicide. The hazard ratio below 1 for religious service attendance can be interpreted to mean that if other variables were constant, individuals who frequently attended religious services were $67 \%$ less likely to die by suicide than those who were not frequent attenders. The survival curves predicting time to death by suicide for frequent $v$. non-frequent religious service attenders 


\begin{tabular}{|c|c|c|c|}
\hline & $\begin{array}{l}\text { Died by suicide } \\
\qquad(n=25)\end{array}$ & $\begin{array}{l}\text { Died by other means } \\
\qquad(n=5251)\end{array}$ & $\begin{array}{c}\text { Alive } \\
(n=14738)\end{array}$ \\
\hline \multicolumn{4}{|l|}{ Dichotomous variables, $n(\%)^{\mathrm{a}}$} \\
\hline Gender (male) & $22(88)$ & $2724(51.81)$ & $6642(45.07)$ \\
\hline Ethnicity (White) & $15(60)$ & 3951 (75.14) & $9754(66.18)$ \\
\hline Marital status (married) & $11(44)$ & 2268 (43.02) & 4701 (31.90) \\
\hline Alcohol use $e^{\text {b }}$ & $5(20)$ & $651(12.38)$ & 1462 (9.92) \\
\hline Marijuana use $e^{c}$ & $4(16)$ & $87(1.65)$ & $995(6.75)$ \\
\hline cocaine use $^{d}$ & $3(12)$ & $57(1.08)$ & $499(3.39)$ \\
\hline Dysthymic disorder & $2(8)$ & $23(0.44)$ & $546(3.70)$ \\
\hline Depression diagnosis & $0(0)$ & $5(0.10)$ & $165(1.12)$ \\
\hline Bipolar type $1 / 2$ diagnosis & $1(4)$ & $7(0.13)$ & $95(0.64)$ \\
\hline Previous suicidal ideation & $4(16)$ & $46(0.87)$ & $1220(8.28)$ \\
\hline Previous suicide attempt & $2(8)$ & $20(0.38)$ & $403(2.73)$ \\
\hline Religious service attendance & $8(32)$ & $2850(54.17)$ & 8306 (56.72) \\
\hline \multicolumn{4}{|l|}{ Continuous variables, mean (s.d.) } \\
\hline Age, years & $38.16(20.24)$ & 70.19 (14.71) & $39.50(16.18)$ \\
\hline Size of household & $4.20(2.83)$ & $2.42(1.65)$ & $3.73(2.07)$ \\
\hline Education, years & $11.04(4.17)$ & $9.70(4.16)$ & $11.08(4.00)$ \\
\hline Poverty index & $2.22(1.88)$ & $2.17(1.65)$ & $2.46(1.80)$ \\
\hline \multicolumn{4}{|l|}{ Frequency of social contact } \\
\hline Telephone calls with friends per week & $10.61(16.36)$ & $9.49(14.29)$ & $11.12(18.11)$ \\
\hline Number of times spent with friends locally & $108.88(127.50)$ & $119.11(140.03)$ & $120.38(132.88)$ \\
\hline Number of times visiting friends out of town & $97.60(134.67)$ & 77.67 (133.57) & $61.54(118.59)$ \\
\hline
\end{tabular}

are shown in Fig. 1. In support of our hypothesis, those who frequently attended religious services were less likely to die by suicide over time than those who did not.

\section{Discussion}

We built on previous research by examining frequent attendance at religious services as a protective factor against completed suicide within a long-term, longitudinal prospective framework. Specifically, we found that those who attended religious services 24 times per year or more were less than half as likely to die by suicide than those who attended less frequently. The protective effect of frequent religious service attendance appears relatively robust in our study, predicting suicide up to 16 years later, even after accounting for other predictors of suicide. Nevertheless, although this finding reached statistical significance, that the

\section{Table 2 Univariate Cox proportional hazard regression analyses of time to death by suicide}

\begin{tabular}{|c|c|c|c|}
\hline & B (s.e.) & Wald $\chi^{2}$ & $\mathrm{HR}(95 \% \mathrm{Cl})$ \\
\hline Gender (male) & $1.63(0.71)$ & $5.23^{* *}$ & $5.11(1.26-20.69)$ \\
\hline Age at interview & $-0.03(0.18)$ & $3.00^{*}$ & $0.97(0.94-1.00)$ \\
\hline Ethnicity (White) & $-0.47(1.06)$ & 0.24 & $0.61(0.08-4.85)$ \\
\hline Marital status (married) & $-0.16(0.70)$ & 0.05 & $0.85(0.22-3.36)$ \\
\hline Size of household & $0.20(0.10)$ & $4.07^{* *}$ & $1.22(1.01-1.48)$ \\
\hline Years of education completed & $0.13(0.12)$ & 1.19 & $1.14(0.90-1.46)$ \\
\hline Poverty index & $0.13(0.17)$ & 0.63 & $1.14(0.82-1.59)$ \\
\hline \multicolumn{4}{|l|}{ Frequency of social contact } \\
\hline Telephone calls with friends & $-0.06(0.02)$ & 0.12 & $0.99(0.96-1.03)$ \\
\hline Number of times spent with friends locally & $-0.01(0.01)$ & 1.72 & $1.00(0.99-1.00)$ \\
\hline Number of times visiting friends out of town & $<0.01(0.01)$ & $<0.01$ & $1.00(0.99-1.00)$ \\
\hline Alcohol use & $0.46(0.56)$ & 0.62 & $1.56(0.50-4.99)$ \\
\hline Marijuana use $^{\mathrm{b}}$ & $1.82(0.75)$ & $5.86^{* *}$ & $6.18(1.41-27.00)$ \\
\hline Cocaine use $^{c}$ & $-0.31(0.68)$ & 0.20 & $0.73(0.19-2.80)$ \\
\hline Dysthymic disorder & $1.10(0.94)$ & 1.35 & $3.00(0.47-19.06)$ \\
\hline Bipolar disorder type $1 / 2$ diagnosis & $0.83(1.06)$ & 0.61 & $2.30(0.29-18.35)$ \\
\hline Previous suicidal ideation & $1.04(0.78)$ & 1.78 & $2.84(0.61-13.13)$ \\
\hline Previous suicide attempt & $1.22(0.92)$ & 1.75 & $3.38(0.56-20.44)$ \\
\hline Religious service attendance & $-2.84(1.08)$ & $6.94^{\star \star}$ & $0.06(0.01-0.54)$ \\
\hline \multicolumn{4}{|c|}{$\begin{array}{l}H R \text {, hazard ratio. } \\
\text { a. Five or more drinks almost every day at any point in lifetime. } \\
\text { b. Marijuana use } \geqslant 100 \text { times in lifetime. } \\
\text { c. Cocaine or crack cocaine use } \geqslant 10 \text { times in lifetime. } \\
{ }^{*} P<0.10,{ }^{*} P<0.05 \text {. }\end{array}$} \\
\hline
\end{tabular}




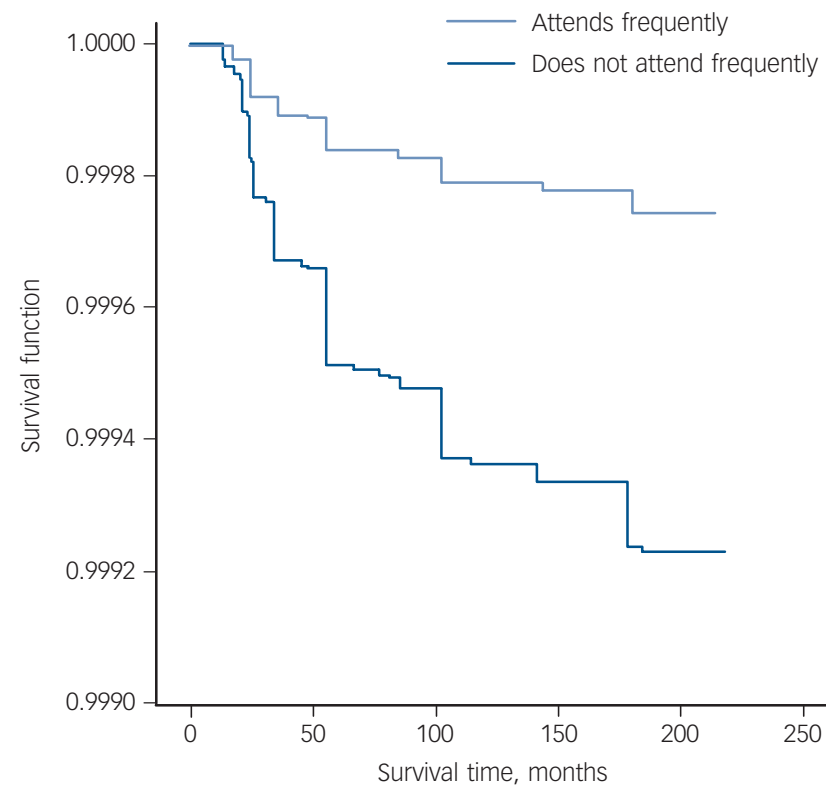

Fig. 1 Survival curves for religious service attendance predicting time to death by suicide.

upper bound of the $95 \%$ confidence interval approached 1.0 suggests the need for some temperance in interpreting this finding. Given the relatively small unweighted number of suicides $(n=25)$ in our sample $(n=20014)$, this is likely to be an issue of power and speaks to the not inconsiderable challenges of conducting longitudinal research on completed suicide. Indeed, although our sample size provided sufficient power to detect medium effects with our observed survival rates, it also limited the ability to detect the significance of small effects (required $n=23312$, observed power 0.74 ). To our knowledge this is the first study within the resilience research literature to prospectively examine death by suicide as an outcome variable. Indeed, none of the studies in a review of protective factors prospectively measured completed suicide. ${ }^{18}$ Moreover, the current study adds to only a handful of others in the general suicide literature that examines prospective predictors of suicide. ${ }^{19-21}$

Religious service attendance is one component of religiousness in general and there is some debate over how religiousness acts as a buffer to suicide. Some theorise that religiousness buffers suicide through the social network it provides, ${ }^{22}$ whereas others theorise that religious prohibitions against suicide may act as a strong and salient deterrent. ${ }^{17,23}$ It should be noted, however, that these two possibilities are not mutually exclusive. Although we did not specifically test a mechanism, our findings are consistent with both theories, and future research determining the manner through which religiousness may exert protective effects against suicide is warranted. Frequent attendance at religious services may be an indicator of consistent exposure to others who provide social support. It may also be an indicator of how much one adheres to the tenets of one's religion, including internalising anti-suicidal beliefs. Moreover, to the extent that religious service attendance - rather than simply religious belief - fosters and is reinforced by a sense of belonging within a community, the current findings are consistent with Joiner's interpersonal theory of suicide, ${ }^{24,25}$ which posits that having a sense of belonging is negatively associated with suicidal desire.

\section{Study limitations}

The limitations of this study involve the use of data that were not explicitly designed for use in psychological research. The NHANES data-set included diagnoses of mood disorders but not of anxiety disorders or post-traumatic stress disorder, which have also been found to be relevant predictors of suicide. ${ }^{19,26}$ The data also did not include the specific religion of the respondents, and thus we were unable to examine whether the effects of religious service attendance on suicide were generalisable across all religions. There are some limitations inherent in dichotomising religious service attendance as a predictor variable. This includes loss of statistical power and a loss of variability within the group (e.g. individuals who attended religious services once a year were grouped with individuals who attended 23 times a year). Although the practice of dichotomising variables is generally inadvisable for these reasons, it is justifiable in cases of highly skewed variables, ${ }^{20,21}$ such as was observed in the case of religious service attendance in our study, to avoid violating assumptions of normality required for conducting survival analysis. Finally, although 25 suicides may seem a relatively low number given the sample size, the figure is in line with the expected suicide rates in the USA between 1988 and 2006. During this time approximately 13.5 per 100000 persons died by suicide per year. ${ }^{27}$ Extrapolating from these figures gives approximately 33 suicides in a group of about 15000 during this time. There is a chance that there were more suicides in this group which were not recorded in the NDI, or were recorded as death by other causes.

It is also particularly interesting that none of the 25 individuals who died by suicide had a diagnosis of depression during the baseline assessment. This might reflect that not all depression diagnoses were ascertained at baseline, or that the individuals who died by suicide developed depression after their NHANES baseline assessment. Suggesting the former possibility, the prevalence of DSM-III major depressive disorder in our sample was $7 \%$. Based on the lifetime prevalence rate of DSM-III major depressive disorder in a similar epidemiological study

\begin{tabular}{|c|c|c|c|}
\hline & B (s.e.) & Wald $\chi^{2}$ & $\mathrm{HR}(95 \% \mathrm{Cl})$ \\
\hline Gender (male) & $1.29(0.62)$ & $4.32^{\star}$ & $3.62(1.01-12.22)$ \\
\hline Age at interview & $-0.02(0.02)$ & 0.41 & $0.98(0.94-1.03)$ \\
\hline Size of household & $0.16(0.10)$ & 2.59 & $1.18(0.97-1.43)$ \\
\hline Marijuana use ${ }^{a}$ & $1.15(0.83)$ & 1.91 & $3.15(0.62-16.07)$ \\
\hline Previous suicide attempt & $0.68(1.03)$ & 0.43 & $1.96(0.26-14.66)$ \\
\hline Religious service attendance & $-1.11(0.58)$ & $3.73^{*}$ & $0.32(0.01-0.99)$ \\
\hline
\end{tabular}


(17\% in the National Comorbidity Survey) and the age at onset distribution of this disorder, ${ }^{28}$ and the mean age of our study sample (38 years), the observed prevalence rate of depression in our sample is slightly lower than would be expected (i.e. approximately $10.6 \%$ ). Suggesting the latter possibility, in a 20 -year prospective study of patients admitted to hospital for suicidal ideation (i.e. individuals likely to be at higher risk of depression than our sample), only 7 of 49 individuals who died by suicide had a diagnosis of depression at baseline. ${ }^{29}$ Nearly all the individuals who died by suicide had a risk factor for depression and subsequent suicide (hopelessness) at baseline. Thus, it is very possible that some of these individuals who died by suicide developed depression after their baseline assessment, as they were already at risk of depression at that time.

\section{Study strengths}

The most notable strength of this study was the use of time to completed suicide as an outcome variable in a large, nationally representative sample. This study is among the few studies in the suicide literature in general and the only study in the protective factors literature specifically to do so. This is important because the main goal of such research is to learn about the processes that can prevent actual suicide. Finally, our study provides important advances over past research, first because it assessed long-term longitudinal prospective predictors of actual death by suicide, and second because it was not reliant on proxy informants, our study variables instead being assessed directly with those who went on to die by suicide.

Evan M. Kleiman, MA, Department of Psychology, George Mason University, Fairfax Virginia; Richard T. Liu, PhD, Department of Psychiatry and Human Behavior, Brown University Alpert Medical School, Bradley Hospital, East Providence, Rhode Island USA

Correspondence: Evan M. Kleiman, Department of Psychology, George Mason University, Mail Stop 3F5, Fairfax, Virginia 22030, USA. Email: ekleiman@gmu.edu

First received 7 Mar 2013, final revision 11 Jun 2013, accepted 18 Jul 2013

\section{References}

1 Hawton K. Sex and suicide. Gender differences in suicidal behaviour. Br J Psychiatry 2000; 177: 484-5.

2 Wilcox HC, Conner KR, Caine ED. Association of alcohol and drug use disorders and completed suicide: an empirical review of cohort studies. Drug Alc Depend 2004; 76 (suppl): s11-9.

3 Dougherty DM, Mathias CW, Marsh DM, Moeller FG, Swann AC. Suicidal behaviors and drug abuse: impulsivity and its assessment. Drug Alcohol Depend 2004; 76 (suppl): s93-105.

4 Hawton K, Sutton L, Haw C, Sinclair J, Harriss L. Suicide and attempted suicide in bipolar disorder: a systematic review of risk factors. J Clin Psychiatry 2005; 66: 693-704.

5 Jamison KR. Suicide and bipolar disorder. J Clin Psychiatry 2000; 61: 47-51.

6 Harris EC, Barraclough B. Suicide as an outcome for mental disorders. A meta-analysis. Br J Psychiatry 1997; 170: 205-28.
7 Joiner TE, Conwell Y, Fitzpatrick KK, Witte TK, Schmidt NB, Berlim MT, et al. Four studies on how past and current suicidality relate even when 'everything but the kitchen sink' is covaried. J Abnorm Psychol 2005; 114: 291-303.

8 Brown GK, Henriques GR, Sosdjan D, Beck AT. Suicide intent and accurate expectations of lethality: predictors of medical lethality of suicide attempts. J Consult Clin Psychol 2004; 72: 1170-4.

9 Mann JJ. A current perspective of suicide and attempted suicide. Ann Intern Med 2002; 136: 302-11.

10 DeJong TM, Overholser JC, Stockmeier CA. Apples to oranges? A direct comparison between suicide attempters and suicide completers. J Affect Disord 2010; 124: 90-7.

11 National Center for Health Statistics. Plan and operation of the Third National Health and Nutrition Examination Survey, 1988-94. Series 1: programs and collection procedures. Vital Health Stat 1994; 32: 1-407.

12 Centers for Disease Control and Prevention, National Center for Health Statistics. NHANES I Epidemiologic Follow-up Survey (NHEFS) Calibration Sample for NDI Matching Methodology. CDC, 2009 (http://www.cdc.gov/ nchs/data/datalinkage/mort_calibration_study.pdf).

13 Fillenbaum GG, Burchett BM, Blazer DG. Identifying a national death index match. Am J Epidemiol 2009; 170: 515-8.

14 Robins LN, Helzer JE, Croughan J, Ratcliff KS. National Institute of Mental Health Diagnostic Interview Schedule. Its history, characteristics, and validity. Arch Gen Psychiatry 1981; 38: 381-9.

15 American Psychiatric Association. Diagnostic and Statistical Manual of Mental Disorders (3rd edn) (DSM-III). APA, 1980.

16 Gillum RF. Frequency of attendance at religious services and cigarette smoking in American women and men: the Third National Health and Nutrition Examination Survey. Prev Med 2005; 41: 607-13.

17 Dervic K, Oquendo MA, Grunebaum MF, Ellis S, Burke AK, Mann JJ. Religious affiliation and suicide attempt. Am J Psychiatry 2004; 161: 2303-8.

18 Johnson J, Wood AM, Gooding P, Taylor PJ, Tarrier N. Resilience to suicidality: the buffering hypothesis. Clin Psychol Rev 2011; 31: 563-91.

19 Hill RM, Castellanos D, Pettit JW. Suicide-related behaviors and anxiety in children and adolescents: a review. Clin Psychol Rev 2011; 31: 1133-44.

20 MacCallum RC, Zhang S, Preacher KJ, Rucker DD. On the practice of dichotomization of quantitative variables. Psychol Methods 2002; 7: 19-40.

21 Streiner DL. Breaking up is hard to do: the heartbreak of dichotomizing continuous data. Can J Psychiatry 2002: 47: 262-6.

22 Durkheim E, Simpson G. Suicide: A Study in Sociology. Simon \& Schuster, 1951.

23 Stack S. The effect of religious commitment on suicide: a cross-national analysis. J Health Soc Behav 1983; 24: 362.

24 Joiner TE, Van Orden KA, Witte TK, Selby EA, Ribeiro J, Lewis R, et al. Main predictions of the interpersonal-psychological theory of suicidal behavior: empirical tests in two samples of young adults. J Abnorm Psychol 2009; 118: 634-46.

25 Joiner TE. Why People Die by Suicide. Harvard University Press, 2005.

26 Krysinska K, Lester D. Post-traumatic stress disorder and suicide risk: a systematic review. Arch Suicide Res 2010; 14: 1-23.

27 Centers for Disease Control and Prevention. National suicide statistics at a glance: Trends in suicide rates among persons ages 10 years and older, by sex, United States, 1991-2009. CDC, 2012 (http://www.cdc.gov/ violenceprevention/suicide/statistics/trends01.html).

28 Kessler RC, McGonagle KA, Zhao S, Nelson CB, Hughes M, Eshleman S, et al. Lifetime and 12-month prevalence of DSM-III-R psychiatric disorders in the United States. Results from the National Comorbidity Survey. Arch Gen Psychiatry 1994; 51: 8-19.

29 Brown GK, Beck AT, Steer RA, Grisham JR. Risk factors for suicide in psychiatric outpatients: a 20-year prospective study. J Consult Clin Psychol 2000; 68: 371-7. 